\title{
Thrombosed saccular aneurysm of a sinus of Valsalva: unusual cause of a mediastinal mass
}

\author{
Peter G Reid, John A Goudevenos, Colin J Hilton
}

\begin{abstract}
A 33 year old man presented with a short history of slight fever and chest pain that was worse on inspiration. An electrocardiogram was consistent with pericarditis. Chest radiography, echocardiography, and computed tomography suggested the presence of a mediastinal tumour. At operation the mass was found to be attached to the right sinus of Valsalva and proved to be a large saccular aneurysm full of laminated thrombus.
\end{abstract}

Aneurysms of sinus of Valsalva often remain undiagnosed until they rupture, usually into the right ventricle or atrium. ${ }^{1}$ Such aneurysms may also present as obstruction of the right ventricular outflow tract, ${ }^{2}$ aortic incompeten$\mathrm{ce}^{3}{ }^{3}$ tricuspid stenosis and incompetence, ${ }^{4}$ coronary artery compression, ${ }^{5}$ and conduction abnormalities. ${ }^{6}$ We describe a patient in whom there was evidence of a mediastinal mass. This proved to be a thrombosed saccular aneurysm originating from the right sinus of Valsalva.

\section{Case report}

33 year old, previously healthy, non-smoking, cardiology registrar referred himself for an electrocardiogram because of a 2 day history of fever and pain behind the sternum and in the right shoulder that was worse on inspiration. The electrocardiogram showed concave upwards elevation of the ST segment in the inferior and anterolateral leads suggestive of pericarditis. On examination he looked well but had a temperature of $37 \cdot 8^{\circ} \mathrm{C}$. His pulse was 70 beats/min, regular, and of normal character and volume. Blood pressure was $110 /$ $70 \mathrm{~mm} \mathrm{Hg}$. The jugular venous pressure was normal as was precordial palpation. Heart sounds were normal and there were no murmurs or rubs. The lungs were clear and the rest of the examination was unremarkable.

A posteroanterior chest radiograph showed an abnormal prominence of the right cardiac border and a lateral film showed an abnormal rounded shadow superimposed on the heart (fig 1). A chest radiograph performed 5 years before, which we were unable to obtain for comparison, had been reported as normal. An echocardiogram showed a large rounded echodense mass adjacent to the aortic root compressing the right atrium (figure 2a). A small rounded echo-free space of uncertain cause was noted within the mass. A small amount of pericardial fluid was seen. The aortic root and valve seemed to be normal. The rest of the examination, including Doppler examination

\footnotetext{
Regional

Cardiothoracic Unit, Freeman Hospital, Newcastle upon Tyne P G Reid J A Goudevenos C J Hilton

Correspondence to Dr Peter G Reid, Regional Cardiothoracic Unit, Freeman Hospital, Freeman Road, High Heaton, Newcastle upon Tyne NE7 7DN.
}

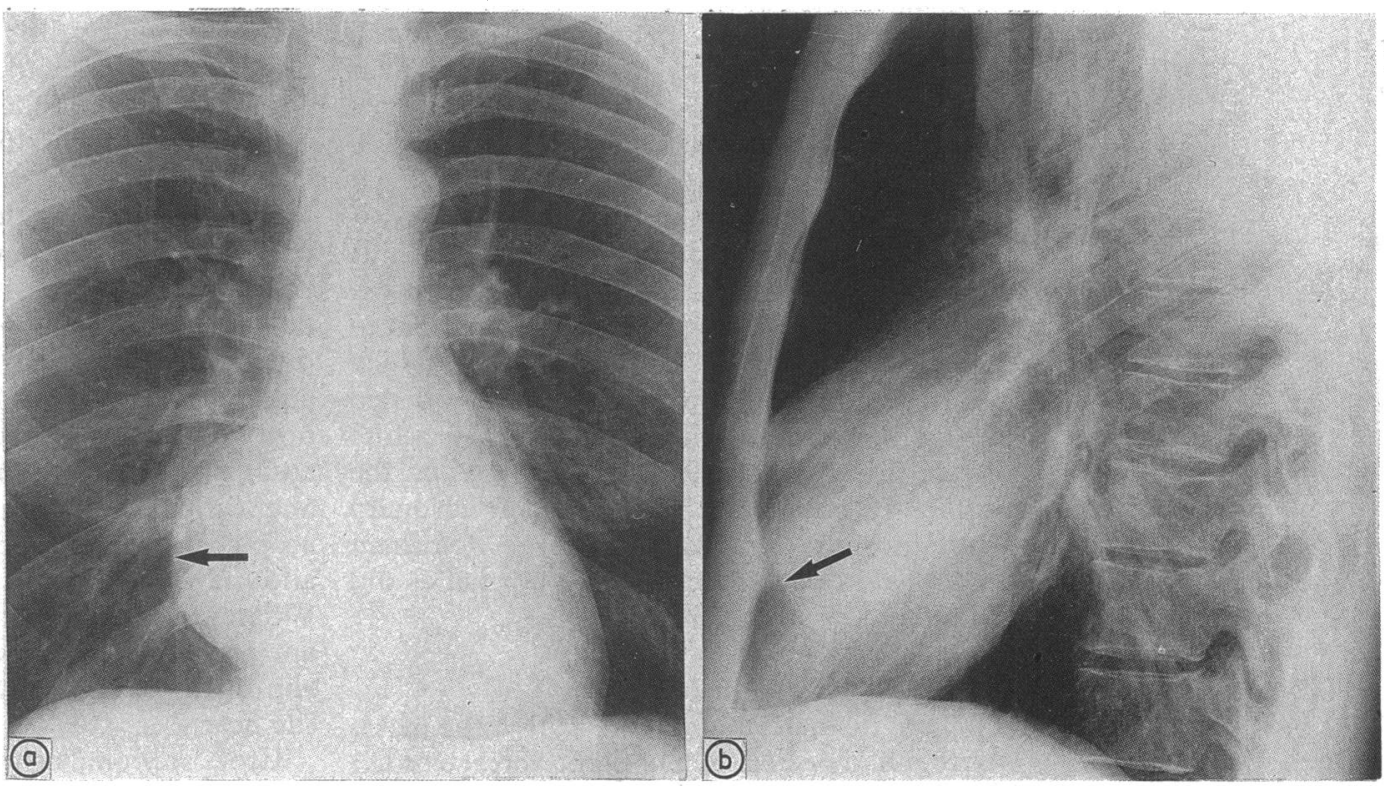

Figure 1 Chest radiographs of (a) posteroanterior view showing prominent right cardiac border (arrow) and (b) lateral view showing abnormal shadow superimposed on heart (arrow). 


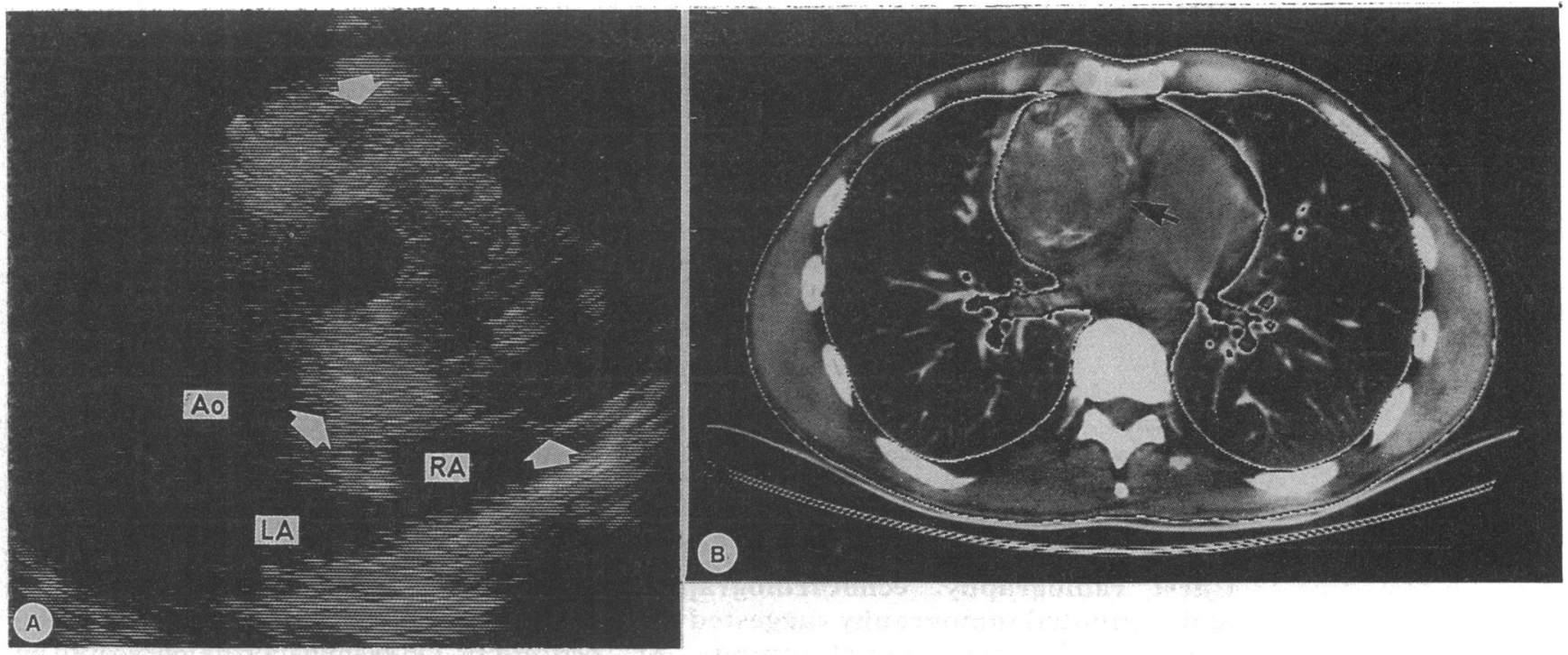

Figure 2 (a) Cross sectional echocardiogram: oblique scan from second right intercostal space showing a mass (arrows) compressing the right atrium. $A$ rounded echo-free space is seen at one pole. Ao, aorta; $L A$, left atrium; $R A$, right atrium. (b) Unenhanced computed tomogram showing the mass (arrow) composed of a mixture of soft tissue and water densities with some calcified areas.

of all cardiac valves, was normal. Laboratory investigations showed a normal full blood count, erythrocyte sedimentation rate, blood urea, creatinine, electrolytes, liver function tests, and immunoglobulin concentrations.

A computed tomogram showed a rounded mass indenting the right side of the heart. It showed a mixture of soft tissue and water densities with several calcified areas (figure 2b). Contrast injection caused considerable enhancement of the nodule on the medial wall of the mass and the margins of the lesion became better defined. A fatty plane seemed to separate most of the mass from the adjacent aortic root and right heart structures. A germ cell tumour was considered to be the most likely diagnosis.

The mass was removed surgically 4 days after presentation.

\section{OPERATIVE FINDINGS}

The mass was situated subepicardially in the atrioventricular groove between the right atrial appendage, aortic root, and right ventricular outflow tract and measured $8 \mathrm{~cm}$ across at its widest point. It seemed to be well encapsulated and was hard and smooth. There was slight pericardial inflammation. The mass was dissected free under cardiopulmonary bypass. It was attached by a $2 \mathrm{~cm}$ diameter pedicle to the right sinus of Valsalva immediately adjacent to the origin of the right coronary artery, which was rudimentary and was unavoidably opened during removal of the mass. A small white plaque resembling atheroma was noted on the wall of the coronary artery. The left coronary artery, which gave rise to a large dominant circumflex vessel, seemed to be normal as did the remainder of the aorta.

\section{PATHOLOGICAL FINDINGS}

A $0.5 \mathrm{~cm}$ diameter hole in the wall of the mass, where it had been attached to the aorta, exuded blood on pressure. Transection showed that the mass had a thin, partly calcified wall and was largely full of laminated thrombus. The appearance suggested a saccular aneurysm. Histological examination showed that the wall was made up of fibrous tissue and smooth muscle with only a little elastic tissue. There was an extensive band of chronic inflammation with infiltration by lymphocytes and plasma cells. Numerous thick-walled blood vessels were seen in this band. There was no evidence of neoplasia.

\section{POSTOPERATIVE COURSE}

The patient's recovery was uneventful and he returned to work after two months. Serological tests for syphilis were negative and random serum cholesterol, measured four months after operation, was $4.0 \mathrm{mmol} / \mathrm{l}$.

\section{Discussion}

Aneurysms arising from a sinus of Valsalva are uncommon and are thought to be most commonly mycotic, arising as a complication of infective endocarditis, or congenital in origin. ${ }^{1}$ Other causes include syphilis, tuberculosis, Marfan and Ehlers-Danlos syndromes, trauma, and other disorders causing aortitis such as ankylosing spondylitis.

The aetiology in the present case is uncertain. Congenital aneurysms usually arise from the lower part of an aortic sinus and enlarge downwards within the heart rather than extend outwards into the pericardial cavity. ${ }^{7}$ Mycotic aneurysms are typically saccular but there was no evidence of an infective cause in the present case. The inflammation seen in the wall of the aneurysm was not accompanied by macroscopic evidence of a more widespread aortitis and may have been a secondary development. Whatever the cause it is remarkable that the aneurysm had attained such a size without rupturing, particularly as it lay on the surface of the heart.

Cross sectional echocardiography was useful in the diagnosis of unruptured aneurysms of a sinus of Valsalva ${ }^{8}$ and computed tomography was considered to be the primary means of 
distinguishing between para-aortic masses and saccular aortic aneurysms although this distinction is difficult when an aneurysm is completely filled with thrombus. ${ }^{9}$ Both investigations were misleading in the present case because such a thrombus was present. In retrospect the echo-free space shown by echocardiography and the enhanced nodule seen on the computed tomogram probably both represented a small lumen in the aneurysm adjacent to its pedicle. Further investigation by aortic angiography might have suggested the correct diagnosis. The importance of angiographic studies of the right and left heart in cases of unusual anterior mediastinal masses that may arise from the heart has been emphasised ${ }^{10}$ but these were not considered to be indicated in our patient because the non-invasive investigations suggested that the mass was a solid tumour.

This case shows that the possibility of a thrombosed vascular aneurysm shold be considered in the differential diagnosis of an apparently solid mediastinal mass.
We thank Dr D S Reid for allowing us to report on a patient under his care.

1 Fishbein MC, Obma R, Roberts WC. Unruptured sinus of Valsalva aneurysm. Am J Cardiol 1975;35:918-22.

2 Kerber RE, Ridges JD, Kriss JP. Unruptured aneurysm of the sinus of Valsalva producing right ventricular outflow obstruction. Am J Med 1972;53:775-83.

3 London SB, London RE. Production of aortic regurgitation by unperforated aneurysm of the sinus of Valsalva. by unperforated aneurysm

4 Gibbs K, Reardon M, Strickman N, et al. Hemodynamic compromise (tricuspid stenosis and insufficiency) caused by an unruptured aneurysm of the sinus of Valsalva. $J A m$ Coll Cardiol 1986;7:1177-81.

5 Chipps HD. Aneurysm of sinus of Valsalva causing coronary occlusion. Arch Pathol 1941;31:627-30.

6 Lee EB, Krieger OJ, Lee NK. Congenital aneurysm of the non-coronary sinus of Valsalva leading to complete heart block: case report. Ann Intern Med 1956;45:525-34.

7 Sakakibara S, Konno S. Congenital aneurysm of the sinus of Valsalva. Anatomy and classification. Am Heart 1962;63:405-24.

8 Lewis BS, Agathangelou NE. Echocardiographic diagnosis of unruptured sinus of Valsalva aneurysm. Am Heart 1984;107:1025-7.

9 Posniak HV, Demos TC, Marsan RE. Computed tomography of the normal aorta and thoracic aneurysms. Semin phy of the normal aorta
Roentgenol 1989;24:7-21.

10 Sethia B, Cotter L. Giant aneurysm of membranous septum. Unusual cause of mediastinal mass. $B r$ Heart $J$ 1981;46:107-9. 